\title{
ELIMINATION THEORY FOR TROPICAL VARIETIES
}

\author{
Bernd Sturmfels and Jenia Tevelev
}

\begin{abstract}
Tropical algebraic geometry offers new tools for elimination theory and implicitization. We determine the tropicalization of the image of a subvariety of an algebraic torus under any homomorphism from that torus to another torus.
\end{abstract}

\section{Introduction}

Elimination theory is the art of computing the image of an algebraic variety under a morphism. Our set-up is as follows. Let $\mathbb{T}^{n}$ be the $n$-dimensional algebraic torus over an algebraically closed field $k$. Given any closed subvariety $X \subset \mathbb{T}^{n}$ and any homomorphism of tori $\alpha: \mathbb{T}^{n} \rightarrow \mathbb{T}^{d}$, our objective is to compute the Zariski closure of the image of $X$ under $\alpha$. Abusing notation, we denote this closure by $\alpha(X)$.

In tropical elimination theory, the varieties $X \subset \mathbb{T}^{n}$ and $\alpha(X) \subset \mathbb{T}^{d}$ are replaced by their tropicalizations. The tropicalization of $X$ is the following subset of $\mathbb{Q}^{n}$ :

$$
\mathcal{T}(X)=\left\{v \in \mathbb{Q}^{n} \mid 1 \notin \operatorname{in}_{v}\left(I_{X}\right)\right\} .
$$

Here $I_{X}$ is the ideal of $X$ in the Laurent polynomial ring $k\left[\mathbb{T}^{n}\right]$, and $\operatorname{in}_{v}\left(I_{X}\right) \subset k\left[\mathbb{T}^{n}\right]$ is the ideal of all initial forms $\operatorname{in}_{v}(f)$ for $f \in I_{X}$. The set $\mathcal{T}(X)$ has the structure

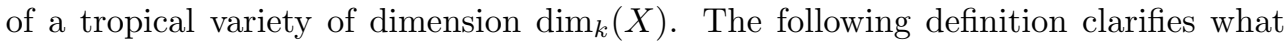
this means for us: an (abstract) tropical variety is a pair $(\mathcal{T}, m)$, where $\mathcal{T} \subset \mathbb{Q}^{n}$ and $m: \mathcal{T}^{0} \rightarrow \mathbb{Z}_{>0}$ is a locally constant function (called multiplicity) which satisfies:

- There exists a pure-dimensional rational polyhedral fan supported on $\mathcal{T}$.

- $\mathcal{T}^{0} \subset \mathcal{T}$ is the open subset of regular points, where $v \in \mathcal{T}$ is called regular if there exists a vector subspace $\mathbb{L}_{v} \subset \mathbb{Q}^{n}$ such that $\mathcal{T}=\mathbb{L}_{v}$ locally near $v$.

- The function $m$ satisfies the balancing condition (see Definition 3.3) for one (and hence for any) fan supported on the set $\mathcal{T}$.

There is generally no canonical (or coarsest) fan structure on $\mathcal{T}(X)$; see Example 5.2. However, $\mathcal{T}(X)$ carries a poset of tropical fans that have desirable algebrogeometric properties $[10,21]$. Example 3.10 shows that not all fans on $\mathcal{T}(X)$ are tropical in the sense of Definition 2.11. A particular tropical fan on $\mathcal{T}(X)$, arising from the Gröbner fan of a homogenization of $I_{X}$, was used for the algorithms in [4].

The multiplicity $m_{v}$ of a point $v$ in $\mathcal{T}(X)^{0}$ can be computed as the sum of the multiplicities of all minimal associate primes of the initial ideal $\operatorname{in}_{v}\left(I_{X}\right)$. This recipe was proposed in $[6, \S 2]$ and it satisfies the balancing condition by [17, §2.5]. A selfcontained derivation of the multiplicities on tropical fans will be given in Section 3.

Returning to our tropical elimination theory, let $\mathcal{T}_{1} \subset \mathbb{Q}^{n}$ and $\mathcal{T}_{2} \subset \mathbb{Q}^{d}$ be abstract tropical varieties. We say that a map $f: \mathcal{T}_{1} \rightarrow \mathcal{T}_{2}$ is generically finite of degree $\delta$ if

- $f$ is the restriction of a linear map $\mathbf{A}: \mathbb{Z}^{n} \rightarrow \mathbb{Z}^{d}$;

- $f$ is surjective and $\operatorname{dim} \mathcal{T}_{1}=\operatorname{dim} \mathcal{T}_{2}$; 
- for any point $w \in \mathcal{T}_{2}^{0}$ such that $f^{-1}(w) \subset \mathcal{T}_{1}^{0}$ and $\left|f^{-1}(w)\right|<\infty$, we have

$$
m_{w}=\frac{1}{\delta} \sum_{v \in f^{-1}(w)} m_{v} \cdot \operatorname{index}\left(\mathbb{L}_{w} \cap \mathbb{Z}^{d}: \mathbf{A}\left(\mathbb{L}_{v} \cap \mathbb{Z}^{n}\right)\right) .
$$

We shall prove the following general result about morphisms of tropical varieties.

Theorem 1.1. Let $\alpha: \mathbb{T}^{n} \rightarrow \mathbb{T}^{d}$ be a homomorphism of tori and let $\mathbf{A}: \mathbb{Z}^{n} \rightarrow \mathbb{Z}^{d}$ be the corresponding linear map of lattices of one-parameter subgroups. Suppose that $\alpha$ induces a generically finite morphism of degree $\delta$ from $X$ onto $\alpha(X)$. Then $\mathbf{A}$ induces a generically finite map of tropical varieties from $\mathcal{T}(X)$ onto $\mathcal{T}(\alpha(X))$.

Remark 1.2. Theorem 1.1 constitutes a refinement of the known identity

$$
\mathcal{T}(\alpha(X))=\mathbf{A}(\mathcal{T}(X)) .
$$

Equation (1.3) appeared in different guises in [21, Proposition 3.1] and in [6, Theorem 3.1]. What is new here is the formula (1.2) for the push-forward of multiplicities.

This paper rests on the foundations of tropical algebraic geometry which were laid by Hacking, Keel, and Tevelev in [10, 21]. We review these foundations in Section 2. A particularly important ingredient is the normal crossing construction in Theorem 2.6 which characterizes tropical varieties in terms of resolutions of singularities.

In Section 3 we discuss multiplicities on tropical varieties, and we highlight the connections to intersection theory on toric varieties. To be precise, we equate the balancing condition with the axiom for Minkowski weights introduced by Fulton and Sturmfels [9]. Theorem 1.1 will then be restated and proved in Theorem 3.12.

In Section 4 we examine the case when $X$ is a generic complete intersection. This case was also studied by Khovanskii and Esterov [13], and we extend some of their results. In our view, the mixed volumes in [13] are best thought of as multiplicities on tropical varieties. Theorem 4.6 gives a formula for the tropical complete intersection $\mathcal{T}(X)$ and its multiplicities in terms of mixed volumes. Our push-forward formula for complete intersections (Corollary 4.8) is particularly interesting when $\alpha(X)$ is a hypersurface, in which case it computes McMullen's mixed fiber polytopes [15].

A special case of elimination is implicitization, which transforms a parametrization of an algebraic variety into its representation as the zero set of polynomials. To model implicitization, we take $X$ to be the graph of the parametrization and $\alpha$ the projection onto the image coordinates. This leads to the implicitization formula in Theorem 5.1. This formula was announced in our paper with $\mathrm{Yu}$ [20]. Its proof is now completed in Section 5 below. Our results generalize the earlier work on $A$ discriminants by Dickenstein, Feichtner and Sturmfels [6], who studied the tropical implicitization problem for Kapranov's Horn uniformization. Software for tropical implicitization, which offers an implementation of Theorem 5.1, is described in [11].

We close the introduction with an explicit example which illustrates Theorem 1.1.

Example 1.3. Let $X$ denote the curve in $\mathbb{T}^{3}$ defined by the two equations

$$
x^{3}+y^{3}+z^{3}=5 \quad \text { and } \quad x^{-2}+y^{-2}+z^{-2}=7 .
$$

We compute the image of $X$ under the map $\alpha: \mathbb{T}^{3} \rightarrow \mathbb{T}^{2},(x, y, z) \mapsto(u, v)$ given by

$$
u=x y z \text { and } v=y z^{2} \quad \text { or, in matrix notation, } \quad \mathbf{A}=\left(\begin{array}{lll}
1 & 1 & 1 \\
0 & 1 & 2
\end{array}\right) .
$$


We demonstrate how one constructs the Newton polygon of the plane curve $\alpha(X) \subset$ $\mathbb{T}^{2}$ prior to knowing its equation. Theorem 4.6 tells us that the tropicalization $\mathcal{T}(X)$ of the space curve $X \subset \mathbb{T}^{3}$ is the one-dimensional fan consisting of the six rays spanned by $\pm(1,1,0), \pm(1,0,1), \pm(0,1,1)$, where each ray has multiplicity 6 . By (1.3), the tropical curve $\mathcal{T}(\alpha(X))$ consists of the rays spanned by the six columns of

$$
6 \cdot \mathbf{A} \cdot\left(\begin{array}{rrrrrr}
1 & 1 & 0 & -1 & -1 & 0 \\
1 & 0 & 1 & -1 & 0 & -1 \\
0 & 1 & 1 & 0 & -1 & -1
\end{array}\right)=\left(\begin{array}{cccccr}
12 & 12 & 12 & -12 & -12 & -12 \\
6 & 12 & 18 & -6 & -12 & -18
\end{array}\right)
$$

We multiply each vector by 6 as a way of recording the information that each ray has multiplicity 6 . To obtain the Newton polygon of the plane curve $\alpha(X)$, we rotate the six vectors by 90 degrees, and concatenate them to form a hexagon with vertices

$$
(0,36),(6,24),(18,12),(36,0),(30,12),(18,24) \text {. }
$$

Each of the 213 lattice points in this hexagon contributes one term to the equation: $u^{36}+v^{36}+15625 u^{6} v^{24}+15625 u^{18} v^{12}-40353607 u^{30} v^{12}-40353607 u^{18} v^{24}+\cdots$. In Section 4 we shall see how the hexagon is constructed as a mixed fiber polytope.

\section{Geometric Tropicalization}

Let $\mathbb{T}$ be an algebraic torus over $k$ and $X \subset \mathbb{T}$ an irreducible closed subvariety. By geometric tropicalization of $X$ we mean the characterization of $\mathcal{T}(X)$ given in $[10,21]$ in terms of constructions of algebraic geometry. We start out by reviewing the connections between the geometric tropicalization and descriptions of $\mathcal{T}(X)$ using valuations of the coordinate ring of $X$ and degenerations of $X$ inside the torus $\mathbb{T}$.

Notation 2.1. Throughout this paper we fix the following notation related to toric varieties. We write $M$ for the lattice of characters of $\mathbb{T}$ and $N:=M^{\vee}$ for the dual lattice. The tropical variety $\mathcal{T}(X)$ will live in $N_{\mathbb{Q}}$. For any fan $\mathcal{F} \subset N_{\mathbb{Q}}$, we denote by $\mathbb{P}(\mathcal{F})$ the corresponding toric variety and by $X(\mathcal{F})$ the closure of $X$ in $\mathbb{P}(\mathcal{F})$.

To streamline our logic, our point of departure will be the valuative definition of $\mathcal{T}(X)$, called the Bieri-Groves set in [7], and not the ideal-theoretic definition (1.1). Let $K / k$ be the field of Puiseux series with parameter $\varepsilon$ and with the valuation

$$
\text { ord }: K \rightarrow \mathbb{Q} \cup\{\infty\}, \quad \alpha \varepsilon^{u}+\text { (higher order terms) } \mapsto u .
$$

Let $K[X]=k[X] \otimes_{k} K$. A ring valuation val $: K[X] \rightarrow \mathbb{Q} \cup\{\infty\}$ is, by definition, any map that can be written as a composition $v \circ f$, where $f: K[X] \rightarrow L$ is a homomorphism to a field and $v: L \rightarrow \mathbb{Q} \cup\{\infty\}$ is a field valuation.

Definition $2.2([2])$. Let $\mathcal{V}(X)$ be the set of valuations of $K[X]$ that restrict to ord on $K$. Any val $\in \mathcal{V}(X)$ specifies an element $[\mathbf{v a l}]$ of $N_{\mathbb{Q}}=\operatorname{Hom}(M, \mathbb{Q})$ by formula

$$
[\mathbf{v a l}](m):=\operatorname{val}\left(\left.m\right|_{X}\right) \text { for any } m \in M \text {. }
$$

We define the tropical variety of $X$ set-theoretically as follows:

$$
\mathcal{T}(X):=\{[\mathbf{v a l}] \mid \operatorname{val} \in \mathcal{V}(X)\} \subset N_{\mathbb{Q}} .
$$

The next two theorems emphasize that not all valuations in $\mathcal{V}(X)$ are needed but we may restrict to valuations defined by germs of curves or to divisorial valuations. 
Theorem 2.3 (Einsiedler-Kapranov-Lind [7]). Any $K$-valued point $\gamma \in X(K)$ defines a valuation on $K[X]$ by the formula $\mathbf{v a l}_{\gamma}(f):=\operatorname{ord} f(\gamma)$ and we have

$$
\mathcal{T}(X)=\left\{\left[\operatorname{val}_{\gamma}\right] \mid \gamma \in X(K)\right\} \subset N_{\mathbb{Q}} .
$$

Theorem 2.4 (Hacking-Keel-Tevelev $[10, \S 2])$. Let $\mathcal{W}(X)$ be the set of divisorial discrete valuations of the function field $k(X)$, i.e. valuations of the form $c \cdot \mathbf{v a l}_{D}$, where $c \in \mathbb{Q}$ and $\mathbf{v a l}_{D}$ is the order of zero-poles along an irreducible divisor $D$ on a normal variety birationally isomorphic to $X$. Then

$$
\mathcal{T}(X)=\{[v] \mid v \in \mathcal{W}(X)\} \subset N_{\mathbb{Q}} .
$$

Instead of $\mathcal{W}(X)$, one can use the set of all discrete valuations of $k(X)$ trivial on $k$.

In [10], Theorem 2.4 is deduced from Theorem 2.6 and Proposition 2.8 using resolution of singularities (or alteration of singularities in prime characteristic). We here give a more straightforward proof which does not require the use of resolution of singularities. Our argument is based on the following characterization of $\mathcal{T}(X)$.

Lemma 2.5 ([21, Lemma 2.2]). Let $w \in N \backslash\{0\}$ and let $\mathcal{F}$ be the one-dimensional fan in $N_{\mathbb{Q}}$ with just one maximal cone $\mathbb{Q}_{\geq 0} w$. Let $D$ the unique toric divisor on $\mathbb{P}(\mathcal{F})$. Then $w$ lies in the tropical variety $\mathcal{T}(X)$ if and only if $D$ intersects $X(\mathcal{F})$.

Proof of Theorem 2.4. Consider any discrete valuation $v$ of the function field $k(X)$ trivial on $k$. We claim that $v$ induces a ring valuation $\mathbf{v}$ of $K[X]$ by the formula

$$
\mathbf{v}\left(\sum_{q \in \mathbb{Q}} a_{q} \varepsilon^{q}\right)=\min _{q \in \mathbb{Q}}\left\{v\left(a_{q}\right)+q\right\},
$$

where $a_{q} \in k[X]$. There exist $p_{1}, \ldots, p_{s} \in K$ and $b_{1}, \ldots, b_{s} \in k[X]$ such that

$$
\sum_{q \in \mathbb{Q}} a_{q}(x) \varepsilon^{q}=\sum_{i=1}^{s} p_{i}(\varepsilon) b_{i}(x),
$$

This identity shows that any $a_{q}$ is a $k$-linear combination of $b_{1}, \ldots, b_{s}$ and therefore $v\left(a_{q}\right) \geq \inf _{i=1}^{s} v\left(b_{i}\right)$. It follows that $\mathbf{v}$ is well-defined. The proof that $\mathbf{v}$ is a valuation goes literally as in [5, VI.10.1]. It follows from (2.6) that the restriction of $\mathbf{v}$ to $K$ coincides with ord, and hence $\mathbf{v} \in \mathcal{V}(X)$. Therefore, $[v]=[\mathbf{v}] \in \mathcal{T}(X)$.

It remains to prove that any point $w \in \mathcal{T}(X)$ has the form $[v]$ for some divisorial discrete valuation $v \in \mathcal{W}(X)$. We use Lemma 2.5 and its notation. Note that $\mathbb{P}(\mathcal{F})=\mathbb{A}_{x_{1}}^{1} \times \mathbb{T}_{x_{2}, \ldots, x_{n}}^{n-1}$ where the coordinates $x_{1}, x_{2}, \ldots, x_{n}$ of $\mathbb{T}=\mathbb{T}^{n}$ are chosen appropriately. By Lemma 2.5, the divisor $D=\left\{x_{1}=0\right\}$ of $\mathbb{P}(\mathcal{F})$ intersects the closure $X(\mathcal{F})$ of $X$. Let $\nu: \tilde{X}(\mathcal{F}) \rightarrow X(\mathcal{F})$ be the normalization and let $Z$ be an irreducible component of $\nu^{-1}(X(\mathcal{F}) \cap D)$. For $i \geq 2$, the coordinate function $x_{i}$ is invertible on $\mathbb{P}(\mathcal{F})$ and hence so is its restriction $\bar{x}_{i}$ to $X(\mathcal{F})$ and its pull-back to $\tilde{X}(\mathcal{F})$. This shows that $\operatorname{val}_{Z}\left(\bar{x}_{2}\right)=\cdots=\operatorname{val}_{Z}\left(\bar{x}_{n}\right)=0$. Since $\nu^{*}\left(\bar{x}_{1}\right)$ vanishes on $Z$, we have $\mathbf{v a l}_{Z}\left(x_{1}\right)=\lambda$ for some positive integer $\lambda$. This implies that $w=\lambda \cdot\left[\mathbf{v a l}_{Z}\right]$ in $N=M^{\vee}$. Now if we set $v:=(1 / \lambda) \cdot \operatorname{val}_{Z}$ then $v \in \mathcal{W}(X)$ and $[v]=w$.

The description of $\mathcal{T}(X)$ using divisorial valuations becomes absolutely explicit if $X$ is smooth and has a known compactification with normally crossing boundary: 
Theorem 2.6 (Hacking-Keel-Tevelev $[10, \S 2])$. Assume that $X$ is smooth and $\bar{X} \supset X$ is any compactification whose boundary $D=\bar{X} \backslash X$ is a divisor with simple normal crossings. Let $D_{1}, \ldots, D_{m}$ denote the irreducible components of $D$, and write $\Delta_{X, D}$ for the simplicial complex on $\{1, \ldots, m\}$ with $\left\{i_{1}, \ldots, i_{l}\right\} \in \Delta_{X, D}$ if and only if $D_{i_{1}} \cap \cdots \cap D_{i_{l}}$ is non-empty. Define $\left[D_{i}\right]:=\left[\mathbf{v a l}_{D_{i}}\right] \in N$, and, for any $\sigma \in \Delta_{X, D}$, let $[\sigma]$ be the cone in $N_{\mathbb{Q}}$ spanned by $\left\{\left[D_{i}\right]: i \in \sigma\right\}$. Then

$$
\mathcal{T}(X)=\bigcup_{\sigma \in \Delta_{X, D}}[\sigma] .
$$

Remark 2.7. The proof in [10] shows that $\mathcal{T}(X)$ is contained in the right hand side of (2.7) if $\bar{X}$ is just normal, without any smoothness or normal crossings conditions. But this containment can be strict: if too many boundary divisors pass through a point then the right hand side can contain cones of dimension greater than $\operatorname{dim} X$. But $\mathcal{T}(X)$ does not contain such cones because $\operatorname{dim} \mathcal{T}(X)=\operatorname{dim} X$.

Later we will discuss ways of weakening the normal crossing assumptions. But our main technique is to use Theorem 2.6 together with the following basic result.

Proposition 2.8 ([21, 3.2]). Consider a commutative diagram of elimination theory

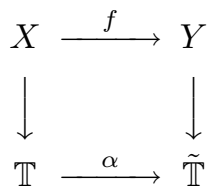

where vertical arrows are closed embeddings in tori, $f$ is dominant, and $\alpha$ is a homomorphism of tori. Let $\tilde{M}$ be the character lattice of $\tilde{\mathbb{T}}$ and $\tilde{N}$ its dual. Then $\alpha$ induces a $\mathbb{Z}$-linear map $\mathbf{A}: N_{\mathbb{Q}} \rightarrow \tilde{N}_{\mathbb{Q}}$ and we have $\mathbf{A}(\mathcal{T}(X))=\mathcal{T}(Y)$ as in (1.3).

Proof. We derive this from Theorem 2.4. Let $y \in \mathcal{T}(Y)$ and let $\tilde{v}$ be a discrete valuation of $k(Y)$ such that $[\tilde{v}]=y$. Let $v$ be a discrete valuation of $k(X)$ such that $\left.v\right|_{k(Y)}=\tilde{v}$. We claim that $\mathbf{A}([v])=y$. We have to check that $\tilde{m}(\mathbf{A}([v]))=\tilde{m}(y)$ for any $\tilde{m} \in \tilde{M}$. This means that $v\left(\alpha^{*} m\right)=\tilde{v}(m)$, which is obvious.

Combining Theorem 2.6 and Proposition 2.8 gives the following corollary.

Corollary 2.9. Suppose that the hypotheses of Proposition 2.8 and Theorem 2.6 hold. Then the tropical variety $\mathcal{T}(Y)$ in $\tilde{N}_{\mathbb{Q}}$ is the union of the cones $\mathbf{A}([\sigma])$ where $\sigma$ runs over all maximal simplices of $\Delta_{X, D}$. The rays $\mathbf{A}\left(\left[D_{i}\right]\right)$ of these cones are the linear forms on $\tilde{M}$ given by $\mathbf{A}\left(\left[D_{i}\right]\right)(\chi):=\operatorname{val}_{D_{i}}(\chi \circ f)$.

Remark 2.10 (Intrinsic Torus [21, $§ 3]$ ). We wish to explain why only monomial maps are considered in our elimination problem. Recall that an irreducible algebraic variety $X$ is called very affine if it is isomorphic to a closed subvariety of an algebraic torus. Intrinsically this means that $X$ is affine and its coordinate algebra $k[X]$ is generated by its units $k[X]^{*}$. In this case any choice of invertible generators $x_{1}, \ldots, x_{n} \in k[X]$ gives a closed embedding $X \subset \mathbb{T}^{n}$. By a theorem of Samuel [16], the group of units $k[X]^{*} / k^{*}$ is finitely generated. Hence any very affine variety is a closed subvariety of a 
canonical intrinsic torus $\mathbb{T}$ with character lattice $k[X]^{*} / k^{*}$. Moreover, any morphism to the algebraic torus $f: X \rightarrow \tilde{\mathbb{T}}$ is the restriction of the homomorphism $\alpha: \mathbb{T} \rightarrow \tilde{\mathbb{T}}$ defined as follows: the pull-back $\alpha^{*}$ is the composition

$$
k[\tilde{\mathbb{T}}]^{*} / k^{*} \rightarrow k[X]^{*} / k^{*} \simeq k[\mathbb{T}]^{*} / k^{*} .
$$

It follows that any elimination problem is monomial, at least in principle.

All of the above results characterize the tropical variety $\mathcal{T}(X)$ only as a subset of a vector space over $\mathbb{Q}$. Next we recall the definition of tropical fans. In Section 3, this will furnish us with a convenient framework for defining multiplicities on $\mathcal{T}(X)$.

Definition 2.11 ([21]). Let $\mathcal{F}$ be any fan in $N_{\mathbb{Q}}$ and $X$ a very affine variety in $\mathbb{T}$. We say that $\mathcal{F}$ is a tropical fan for $X$ if $X(\mathcal{F})$ is proper and the multiplication map

$$
\Psi: \mathbb{T} \times X(\mathcal{F}) \rightarrow \mathbb{P}(\mathcal{F})
$$

is flat and surjective. Note that this property depends on both $X$ and $\mathcal{F}$.

Theorem 2.12 ([21, 1.2, 2.5]). Tropical fans have the following properties:

(1) If $\mathcal{F}$ is a tropical fan (for $X$ ) then the support of $\mathcal{F}$ equals the tropical variety $\mathcal{T}(X)$. Any fan $\mathcal{F}$ supported on $\mathcal{T}(X)$ has a tropical refinement $\mathcal{F}^{\prime}$.

(2) If $\mathcal{F}$ is tropical and $\mathcal{F}^{\prime}$ is any refinement of $\mathcal{F}$ then $\mathcal{F}^{\prime}$ is tropical as well.

(3) Let $\Gamma \subset \mathcal{F}$ be a maximal-dimensional cone and let $Z \subset \mathbb{P}(\mathcal{F})$ be the corresponding toric stratum. If $\mathcal{F}$ is tropical then $\operatorname{dim} X(\mathcal{F}) \cap Z=0$. If, moreover, $\mathbb{P}(\mathcal{F})$ is smooth then $X(\mathcal{F})$ is Cohen-Macaulay at any point of $X(\mathcal{F}) \cap Z$.

The notion of a tropical fan is a very flexible generalization of the Gröbner fan structure on $\mathcal{T}(X)$ used in $[4,6,18]$. We briefly review this connection. To keep the notation simple, we assume from now on that the normalizer of $X$ in $\mathbb{T}$ is trivial.

Theorem 2.13 ([21, 1.7]). Let $\mathcal{G}$ be any complete fan in $N_{\mathbb{O}}$. Consider the action of $\mathbb{T}$ on the Hilbert scheme of $\mathbb{P}(\mathcal{G})$ by the formula $t \cdot[S]=\left[t^{-1} S\right]$ for any subscheme $S \subset \mathbb{P}(\mathcal{G})$. The normalization of the orbit closure $\overline{\mathbb{T} \cdot[X(\mathcal{G})]}$ in the Hilbert scheme is a toric variety for $\mathbb{T}$. Let $\tilde{\mathcal{F}}$ be the complete fan of this toric variety. The intersection of $\tilde{\mathcal{F}}$ with $\mathcal{T}(X)$ is a subfan of $\tilde{\mathcal{F}}$, and that subfan is a tropical fan for $X$.

Now we relate Theorem 2.13 to Gröbner fans using the Cox coordinate ring. Let $m$ be the number of rays of $\mathcal{G}$ and let $f: \mathbb{Z}^{m} \rightarrow N$ be a linear map that sends the $i$-th basis vector $e_{i}$ to the generator of the $i$-th ray of $\mathcal{G}$. This induces a surjective homomorphism $\mathbb{T}^{m} \rightarrow \mathbb{T}$. Consider the standard embedding $\mathbb{T}^{m} \subset \mathbb{A}^{m}$ and let $U \subset \mathbb{A}^{m}$ be a $\mathbb{T}^{m}$-toric open subset obtained by throwing away coordinate subspaces $\left\langle e_{i}: i \in I\right\rangle$ for each collection of boundary divisors $\left\{D_{i}: i \in I\right\}$ with empty intersection. Then we have a morphism of toric varieties $\pi: U \rightarrow \mathbb{P}(\mathcal{G})$ extending the homomorphism $\mathbb{T}^{m} \rightarrow \mathbb{T}$. Let $R=k\left[\mathbb{A}^{m}\right]$, the Cox ring of $\mathbb{P}(\mathcal{G})$. Let $\hat{X} \subset \mathbb{A}^{m}$ be the closure of $\pi^{-1}(X)$ and let $I \subset R$ be its ideal. The Gröbner fan $\hat{\mathcal{F}} \subset \mathbb{Q}^{m}$ is, by definition, supported on the first octant $\mathbb{Q}_{\geq 0}^{m}$, and two vectors $w_{1}$ and $w_{2}$ are in the relative interior of the same cone if and only if the initial ideals $\operatorname{in}_{w_{1}}(I)$ and $\operatorname{in}_{w_{2}}(I)$ in $R$ are equal. It is not hard to prove (using the fact that initial ideals compute flat limits of one-parameter subgroups) that $f$ induces the map of fans $\hat{\mathcal{F}} \rightarrow \tilde{\mathcal{F}}$. Moreover, $\sigma=f^{-1}(f(\sigma))$ for any cone $\sigma \in \hat{\mathcal{F}}$. Since a refinement of a tropical fan is tropical (Theorem 2.12), it 
is possible to define a tropical fan supported on $\mathcal{T}(X)$ by taking images of cones in $\hat{\mathcal{F}}$ that intersect (= are contained in) $\mathcal{T}(X)$. It is this fan that was "the tropical variety" in the earlier papers $[4,6,18]$. Note that this fan can be finer than the fan $\mathcal{F}$ defined in Theorem 2.13 because initial ideals $\operatorname{in}_{w} I$ can have embedded components supported on the unstable locus $\mathbb{A}^{m} \backslash U$.

\section{Push-Forward of Multiplicities}

We now turn our attention to the multiplicities attached to a tropical variety. A tropical fan structure will used to define multiplicities, but that definition turns out to be equivalent to the one given in the introduction (see Corollary 3.15) and thus independent of the particular choice of tropical fan. Our main result is Theorem 1.1, which describes what happens to the multiplicities under a morphism $\alpha: \mathbb{T}^{n} \rightarrow \mathbb{T}^{d}$.

We retain the notation from Section 2, in particular, $X$ is a closed subvariety of an algebraic torus $\mathbb{T}$ and $\mathcal{T}(X) \subset N_{\mathbb{Q}}$ is its tropicalization. If $\mathcal{F} \subset N_{\mathbb{Q}}$ is any fan, with corresponding toric variety $\mathbb{P}(\mathcal{F})$, then $X(\mathcal{F})$ denotes the closure of $X$ in $\mathbb{P}(\mathcal{F})$.

Definition 3.1. Suppose that $\mathcal{F}$ is a tropical fan for $X$. Let $\Gamma \subset \mathcal{F}$ be a maximaldimensional cone (i.e. of dimension $\operatorname{dim}(X))$ and let $Z \subset \mathbb{P}(\mathcal{F})$ be the torus orbit that corresponds to $\Gamma$. Note that the scheme $X(\mathcal{F}) \cap Z$ is 0 -dimensional by Theorem 2.12 . We define the multiplicity $m_{\Gamma}$ of the cone $\Gamma$ to be the length of the scheme $X(\mathcal{F}) \cap Z$.

Lemma 3.2. Let $\mathcal{F}$ be any tropical fan for $X$.

(1) If $\mathbb{P}(\mathcal{F})$ is smooth then $m_{\Gamma}$ equals the intersection number $\operatorname{deg}([Z] \cdot[X(\mathcal{F})])$.

(2) If $\mathcal{F}^{\prime}$ is any fan that refines the given tropical fan $\mathcal{F}$ then $m_{\Gamma^{\prime}}=m_{\Gamma}$ for any pair of maximal-dimensional cones $\Gamma^{\prime}$ in $\mathcal{F}^{\prime}$ and $\Gamma$ in $\mathcal{F}$ such that $\Gamma^{\prime} \subseteq \Gamma$.

Proof. Statement (1) follows from [8, Proposition 7.1]. Recall that, in general, the intersection number of a proper intersection can be less than the length of an intersection subscheme. However, such bad intersections do not happen in our case since $X(\mathcal{F})$ is Cohen-Macaulay at any point of $X(\mathcal{F}) \cap Z$, by Theorem $2.12(3)$.

To prove (2), let $Z \subset \mathbb{P}(\mathcal{F})$ and $Z^{\prime} \subset \mathbb{P}\left(\mathcal{F}^{\prime}\right)$ be the torus orbits that correspond to $\Gamma$ and $\Gamma^{\prime}$ respectively. We consider the commutative diagram

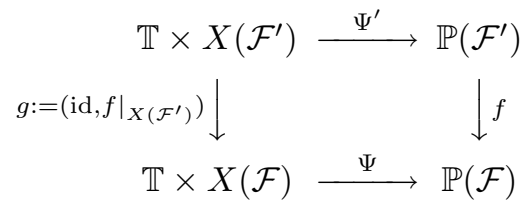

where $\Psi$ and $\Psi^{\prime}$ are multiplication morphisms (2.9), and $f$ is the proper morphism of toric varieties that corresponds to the refinement of fans. This is a fiber diagram by [21, Proposition 2.5]. Let $p^{\prime} \in Z^{\prime}$ be any point. Since $\Psi$ is flat, we have

$$
g_{*}\left(\Psi^{\prime *}\left(\left[p^{\prime}\right]\right)\right)=\Psi^{*}\left(f_{*}\left(\left[p^{\prime}\right]\right)\right),
$$

where we use push-forward and flat pull-back of cycles as in $[8,1.4$ and 1.7]. Let $p=f\left(p^{\prime}\right)$, and let $H$ and $H^{\prime}$ denote the stabilizers in $\mathbb{T}$ of $p$ and $p^{\prime}$ respectively. Since $\mathbb{P}\left(\mathcal{F}^{\prime}\right)$ and $\mathbb{P}(\mathcal{F})$ are normal, the subgroups $H$ and $H^{\prime}$ are subtori of $\mathbb{T}$. Since $\Gamma$ and $\Gamma^{\prime}$ have the same dimension, namely $\operatorname{dim}(X)$, we conclude that $H=H^{\prime}$. 
It follows from the $\mathbb{T}$-invariance of the multiplication map $\Psi$ that the schemetheoretic fiber $(\Psi)^{-1}(p)$ is isomorphic to $H \times(X(\mathcal{F}) \cap Z)$. This product is a torus times a zero-dimensional scheme. Similarly, the scheme-theoretic fiber $\left(\Psi^{\prime}\right)^{-1}\left(p^{\prime}\right)$ is isomorphic to $H \times\left(X\left(\mathcal{F}^{\prime}\right) \cap Z^{\prime}\right)$, with the same torus $H$. The identity (3.10) implies that the length $m_{\Gamma}$ of $X(\mathcal{F}) \cap Z$ coincides with the length $m_{\Gamma^{\prime}}$ of $X\left(\mathcal{F}^{\prime}\right) \cap Z^{\prime}$.

Definition 3.3 (Fulton-Sturmfels [9]). Given a rational polyhedral cone $\sigma \subset N_{\mathbb{Q}}$, we write $N_{\sigma}$ for the sublattice of $N$ generated by $\sigma \cap N$. For a facet $\tau$ of $\sigma$ let $n_{\sigma, \tau}$ be any representative in $\sigma$ for the generator of the 1 -dimensional lattice $N_{\sigma} / N_{\tau}$. A multiplicity function $m_{\sigma}$ defined on $k$-dimensional cones of some fan $\mathcal{F}$ is said to satisfy the balancing condition if, for any cone $\tau$ of $\mathcal{F}$ of dimension $k-1$, we have

$$
\sum_{\sigma \supset \tau} m_{\sigma} \cdot n_{\sigma, \tau} \in N_{\tau}
$$

Here the sum over all $k$-dimensional cones $\sigma$ in $\mathcal{F}$ that contain $\tau$.

Corollary 3.4. If $\mathcal{F}$ is a tropical fan for $X$ whose toric variety $\mathbb{P}(\mathcal{F})$ is smooth then the multiplicity function $m_{\sigma}$ in Definition 3.1 satisfies the balancing condition.

Proof. Let $k=\operatorname{dim} X$. Since we wish to cite [9], we fix a complete strictly simplicial fan $\mathcal{G}$ that contains $\mathcal{F}$ as a subfan. Since $X(\mathcal{F})$ is proper, $X(\mathcal{F})$ does not intersect toric strata parametrized by cones in $\mathcal{G} \backslash \mathcal{F}$. We set $m_{\sigma}=0$ for $k$-dimensional cones $\sigma$ of $\mathcal{G}$ that are not in $\mathcal{F}$. Lemma $3.2(1)$ implies that the multiplicities $m_{\sigma}$ determine a class in the operational Chow cohomology $A^{k}(\mathbb{P}(\mathcal{G})$ ), representing the intersection with $X(\mathcal{F})$. Therefore $m_{\sigma}$ satisfies the balancing condition by [9, Theorem 2.1].

Remark 3.5. From this perspective, a tropical variety is the same as an operational Chow cohomology class on an appropriate toric variety. See also [12, Remark 1.7].

Lemma 3.6. Suppose $p \in \mathcal{T}(X)$ is a regular point (see Introduction) and suppose $\mathcal{F}$ is a fan supported on $\mathcal{T}(X)$ and endowed with a multiplicity function $m_{\sigma}$ that satisfies the balancing condition. Then $m_{\sigma}$ is constant on all cones $\sigma$ containing $p$.

Proof. Restricting to $\mathbb{L}_{p}$ and taking the link $\mathcal{G}$ in $\mathcal{F}$ of the cone containing $p$ in its relative interior, we reduce to the following statement: If $\mathcal{G}$ is a complete fan and its top-dimensional cones are endowed with multiplicities that satisfy the balancing condition then these multiplicities are constant. This holds because the operational Chow cohomology group $A^{0}$ of a complete toric variety $\mathbb{P}(\mathcal{G})$ has rank one.

Definition 3.7. The multiplicity of a regular point $p \in \mathcal{T}(X)$ as the multiplicity $m_{\sigma}$ of any top-dimensional cone $\sigma$ of any tropical fan that contains $p$ in its closure.

Corollary 3.8. The multiplicity is a well-defined locally constant function on the open subset of regular points of $\mathcal{T}(X)$. For any (not necessarily tropical) fan $\mathcal{F}$ supported on $\mathcal{T}(X)$, the induced multiplicity function satisfies the balancing condition. 
Proof. This follows from Lemma 3.6, Corollary 3.4, Lemma 3.2, and the fact that any two tropical fans have a common strictly simplicial refinement.

The balancing condition implies that many complete fans can play the role of the Gröbner fans in Theorem 2.13 when computing tropical varieties set-theoretically:

Proposition 3.9. Let $\mathcal{G} \subset N_{\mathbb{Q}}$ be any complete fan such that $X(\mathcal{G})$ does not intersect toric strata of $\mathbb{P}(\mathcal{G})$ of codimension greater than $k=\operatorname{dim} X$. Then $\mathcal{T}(X)$ equals the union of all $k$-dimensional cones in $\mathcal{G}$ whose toric strata intersect $X(\mathcal{G})$.

Proof. Let $\mathcal{F}$ denote the subfan of $\mathcal{G}$ consisting of all cones whose toric strata intersect $X(\mathcal{G})$. By Theorem 2.12, there exists a tropical fan $\tilde{\mathcal{F}}$ for $X$ which admits a map of fans $\tilde{\mathcal{F}} \rightarrow \mathcal{G}$. By [21, Lemma 2.2], the support $|\tilde{\mathcal{F}}|=\mathcal{T}(X)$ is contained in $|\mathcal{F}|$ and intersects the relative interior of every cone in $\mathcal{F}$. Let $C$ be a $k$-dimensional cone in $\mathcal{F}$ and let $\tilde{\mathcal{F}}_{C}$ be the set of cones in $\tilde{\mathcal{F}}$ contained in $C$. Note that $\tilde{\mathcal{F}}_{C}$ is a $k$-dimensional fan. We claim that its support $\left|\tilde{\mathcal{F}}_{C}\right|$ equals $C$. Arguing by contradiction, suppose that it is not. Then there exists a $k$-dimensional cone $\sigma$ in $\tilde{\mathcal{F}}_{C}$ and a facet $\tau$ of $\sigma$ such that $\tau$ intersects the interior of $C$ and lies in the boundary of $\left|\tilde{\mathcal{F}}_{C}\right|$. But this contradicts the balancing condition, and we conclude $|\mathcal{F}|=\mathcal{T}(X)$.

Example 3.10. The fan $\mathcal{F}$ in the proof of Proposition 3.9 is not necessarily tropical. We construct a variety $X \subset \mathbb{T}$ that admits a non-tropical fan $\mathcal{F}$ supported on $\mathcal{T}(X)$. Consider a projective variety $Y \subset \mathbb{P}^{r-1}$ which is not locally Cohen-Macaulay at some point $p \in Y$. Then $k:=\operatorname{dim} X \geq 2$. Let $H_{1}, \ldots, H_{k} \subset \mathbb{P}^{r-1}$ be general hyperplanes passing through $p$ and let $H_{k+1}, \ldots, H_{r} \subset \mathbb{P}^{r-1}$ be general hyperplanes. The intersection of $\cap_{i \in I} H_{i}$ with $Y$ is non-empty if $|I|=k$ and empty if $|I|>k$. Then $X:=Y \backslash\left\{H_{1}, \ldots, H_{r}\right\}$ is a very affine variety with intrinsic torus $\mathbb{T}=\mathbb{P}^{r-1} \backslash\left\{H_{1}, \ldots, H_{r}\right\}$. Let $\mathcal{F}$ be a fan obtained by taking the $k$-skeleton of the fan of $\mathbb{P}^{r-1}$. By Proposition 3.9, $\mathcal{F}$ is supported on $\mathcal{T}(X)$. If $\mathcal{F}$ is a tropical fan then $Y$ has to be Cohen-Macaulay at $p$ by Theorem $2.12(3)$. Therefore $\mathcal{F}$ is not tropical.

Remark 3.11. This example has to be compared with [10, Theorem 1.9] which concerns the (Mori-theoretically significant) class of hübsch very affine varieties. If $X$ is hübsch then any fan supported on $\mathcal{T}(X)$ is tropical, and there exists a coarsest fan supported on $\mathcal{T}(X)$. The latter property also fails in general, see Example 5.2.

We now explain how multiplicities behave under a map of tropical varieties as discussed in the Introduction. Our setting is that of the commutative diagram (2.8), where vertical arrows are closed embeddings in algebraic tori, $f: X \rightarrow Y$ is dominant, and $\alpha$ is the homomorphism of tori specified by a $\mathbb{Z}$-linear map $\mathbf{A}: N_{\mathbb{Q}} \rightarrow \tilde{N}_{\mathbb{Q}}$. By Proposition 2.8, we have $A(\mathcal{T}(X))=\mathcal{T}(Y)$, which is the identity in (1.3). To prove Theorem 1.1, it suffices to prove the following more technical statement.

Theorem 3.12. Let $\mathcal{F}$ be a fan in $N_{\mathbb{Q}}$ whose support equals $\mathcal{T}(X)$, and let $\tilde{\mathcal{F}}$ be a fan in $\tilde{N}_{\mathbb{Q}}$ whose support equals $\mathcal{T}(Y)$. We further assume the following conditions:

- The map $f$ is generically finite of degree $\delta($ hence $\operatorname{dim} \tilde{\mathcal{F}}=\operatorname{dim} \mathcal{F})$.

- The fan $\mathcal{F}$ is tropical for $X$ and the fan $\tilde{\mathcal{F}}$ is tropical for $Y$.

- For any cone $\Gamma$ of $\mathcal{F}$, the image $\mathbf{A}(\Gamma)$ is a union of cones of $\tilde{\mathcal{F}}$. 
Then the multiplicity $m_{\Pi}$ of any maximal-dimensional cone $\Pi$ of $\tilde{\mathcal{F}}$ satisfies

$$
m_{\Pi}=\frac{1}{\delta} \cdot \sum_{\Gamma \in \mathcal{F}: \mathbf{A}(\Gamma) \supseteq \Pi} m_{\Gamma} \cdot \operatorname{index}(\Gamma, \Pi),
$$

where $\operatorname{index}(\Gamma, \Pi)$ denotes the index of the sublattice of $\tilde{N}$ generated by the semigroup $\mathbf{A}(\Gamma \cap N)$ inside the sublattice generated by the semigroup $\Pi \cap \tilde{N}$.

Proof. Let $k:=\operatorname{dim}(X)$. By Lemma $3.2(2)$ we can replace $\tilde{\mathcal{F}}$ by any refinement. We will thus assume from now on that $\tilde{\mathcal{F}}$ is strictly simplicial, i.e. $\mathbb{P}(\tilde{\mathcal{F}})$ is smooth.

Our next goals are to make $\mathbb{P}(\mathcal{F})$ smooth and the morphism $\mathbb{P}(\mathcal{F}) \rightarrow \mathbb{P}(\tilde{\mathcal{F}})$ flat. These goals are incompatible but the following lemma provides a compromise.

Lemma 3.13. There exists a strictly simplicial refinement $\mathcal{F}^{\prime}$ of $\mathcal{F}$ such that

- $\mathbf{A}: \mathcal{F}^{\prime} \rightarrow \tilde{\mathcal{F}}$ is a map of fans, and

- if $\Gamma$ is any $k$-dimensional cone of $\mathcal{F}^{\prime}$ and $\Pi$ is the minimal cone of $\mathcal{F}$ that contains $\mathbf{A}(\Gamma)$ then either $\operatorname{dim} \Pi<\operatorname{dim} \Gamma$ or $\Pi=\mathbf{A}(\Gamma)$.

Proof of Lemma 3.13. We construct $\mathcal{F}^{\prime}$ in two steps. First we define a refinement $\mathcal{F}^{\prime \prime}$ as follows. For any cone in $\Gamma$ (of any dimension), the image $\mathbf{A}(\Gamma)$ is a union of cones in $\tilde{\mathcal{F}}$. We subdivide $\Gamma$ by preimages of these cones. Since $\mathcal{F}$ is a fan, this subdivision yields a fan $\mathcal{F}^{\prime \prime}$. Moreover, if $\Gamma \in \mathcal{F}^{\prime \prime}$ and $\operatorname{dim} \Gamma=\operatorname{dim} \mathbf{A}(\Gamma)$ then $\Gamma$ is strictly simplicial since $\mathbf{A}(\Gamma)$ is. To finish the construction of $\mathcal{F}^{\prime}$, it remains to subdivide all cones $\Gamma \in \mathcal{F}^{\prime \prime}$ with $\operatorname{dim} \Gamma>\operatorname{dim} \bar{g}(\Gamma)$ into strictly simplicial subcones. But we have to make sure that the collection of new cones is still a fan. Arguing by induction on $\operatorname{dim} \Gamma$, this construction reduces to the following fact which is well-known in geometric combinatorics: if $\Gamma$ is a rational polyhedral cone and $\partial \mathcal{H}$ is a strictly simplicial fan supported on the boundary $\partial \Gamma$, then there is a strictly simplicial fan $\mathcal{H}$ supported on $\Gamma$ such that the restriction of $\mathcal{H}$ to $\partial \Gamma$ equals $\partial \mathcal{H}$.

By Lemma $3.2(2)$, to prove (3.11) it suffices to prove the analogous formula

$$
m_{\Pi}=\frac{1}{\delta} \cdot \sum_{\Gamma \in \mathcal{F}^{\prime}: \mathbf{A}(\Gamma)=\Pi} m_{\Gamma} \cdot \operatorname{index}(\Gamma, \Pi)
$$

for any $d$-dimensional cone $\Pi$ of $\tilde{\mathcal{F}}$. To simplify notation we redenote $\mathcal{F}^{\prime}$ by $\mathcal{F}$.

Consider the smooth toric varieties $\mathbb{P}(\mathcal{F})$ and $\mathbb{P}(\tilde{\mathcal{F}})$. The map of fans $\mathbf{A}: \mathcal{F} \rightarrow \tilde{\mathcal{F}}$ defines a morphism $\alpha: \mathbb{P}(\mathcal{F}) \rightarrow \mathbb{P}(\tilde{\mathcal{F}})$ that extends the homomorphism of tori $\alpha$ : $\mathbb{T} \rightarrow \tilde{\mathbb{T}}$. The $d$-dimensional cone $\Gamma \subset \tilde{\mathcal{F}}$ defines a torus orbit $\tilde{Z}$ of codimension $d$ in $\mathbb{P}(\tilde{\mathcal{F}})$. Our hypotheses imply that the fiber $\alpha^{-1}(\tilde{Z})$ is the disjoint union of torus orbits $Z_{1}, \ldots, Z_{r}$ of codimension $d$ in $\mathbb{P}(\mathcal{F})$. These orbits correspond to the cones $\Gamma_{1}, \ldots, \Gamma_{r}$ in $\mathcal{F}$ that satisfy $\mathbf{A}\left(\Gamma_{i}\right)=\Pi$. Moreover, it is known (see e.g. [9, Prop. 2.7]) that the multiplicity of the scheme $\alpha^{-1}(\tilde{Z})$ along $Z_{i}$ is $\operatorname{index}\left(\Gamma_{i}, \Pi\right)$. 
By Theorem 2.12, the closure $X(\mathcal{F})$ of $X$ in $\mathbb{P}(\mathcal{F})$ is Cohen-Macaulay along the subscheme $W:=\alpha^{-1}(\tilde{Z}) \cap X(\mathcal{F})$. Therefore, we have a fiber diagram

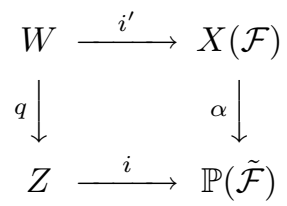

where $i$ and $i^{\prime}$ are regular embeddings of codimension $d$. By Lemma 3.2 we have $m_{\Pi}=\operatorname{deg}([X(\tilde{\mathcal{F}})] \cdot[\tilde{Z}])$. Since the morphism $\alpha$ is proper, the push-forward property of [8, Theorem 6.2 (a) and Remark 6.2.2] implies that

$$
m_{\Pi}=\operatorname{deg}\left(\frac{1}{\delta} \alpha_{*}([X(\mathcal{F})]) \cdot[\tilde{Z}]\right)=\frac{1}{\delta} \operatorname{deg}\left(q_{*}([W])\right)=\frac{1}{\delta} \operatorname{deg}[W] .
$$

From the irreducible decomposition of $\alpha^{-1}(\tilde{Z})$ discussed above, we obtain

$$
\operatorname{deg}[W]=\sum_{i=1}^{r} \operatorname{index}\left(\Gamma_{i}, \Pi\right) \cdot \operatorname{deg}\left([\tilde{X}] \cdot Z_{i}\right)=\sum_{i=1}^{r} \operatorname{index}\left(\Gamma_{i}, \Pi\right) \cdot m_{\Gamma_{i}} .
$$

This completes the proof of Theorem 3.12, and hence also of Theorem 1.1.

Example 3.14. The special case of our construction when $X$ is defined by linear equations appears in $[6, \S 3]$. Here $\mathcal{T}(X)$ is the Bergman fan of a matroid. This is applied in $[6, \S 4]$ to tropicalize Kapranov's Horn uniformization of the A-discriminant.

At this point we wish to note that the definition of multiplicity used here (Definitions 3.1 and 3.7) agrees with the formulation in the Introduction (and in [6, 20]):

Corollary 3.15 ([6, Remark 2.1]). Let $X$ be a subvariety of a torus $\mathbb{T}$ and $I_{X}$ its ideal in $k[\mathbb{T}]$. If $w$ is any regular point in the tropical variety $\mathcal{T}(X)$ then $m_{\sigma}$ equals the sum of multiplicities of all minimal associate primes of the initial ideal $i n_{w}\left(I_{X}\right)$.

Proof. This can be derived from the proof of part (2) in Lemma 3.2.

Example 3.16. Consider an irreducible hypersurface $X$ in $\mathbb{T}$ with principal ideal $I_{X}=\langle G\rangle$. The Laurent polynomial $G$ is unique up to multiplication by a unit. Its Newton polytope $Q \subset M_{\mathbb{Q}}$ is unique up to translation. For any $w \in N_{\mathbb{Q}}$, $\operatorname{in}_{w}(G)$ is the sum of all terms in $G$ that belong the face of $Q$ at which $w$ is minimized. This face is denoted face ${ }_{w}(Q)$, and we have $\operatorname{in}_{w}\left(I_{X}\right)=\left\langle\operatorname{in}_{w}(G)\right\rangle$. The tropical hypersurface $\mathcal{T}(X)$ is the set of $w \in N_{\mathbb{Q}}$ such that face ${ }_{w}(Q)$ has dimension $\geq 1$, i.e. $\mathcal{T}(X)$ is a normal fan of $Q$ with top-dimensional cones removed. A point $w \in \mathcal{T}(X)$ is regular when face $_{w}(Q)$ is an edge. In this case we regard $\operatorname{in}_{w}(G)$ as a univariate polynomial. Its roots correspond to minimal associate primes of $\operatorname{in}_{w}\left(I_{X}\right)$. Counting multiplicities, the number $m_{\sigma}$ of roots equals the number of lattice points on the edge face ${ }_{w}(Q)$.

Remark 3.17. In Example 3.16, the Newton polytope of $Q$ can be recovered (up to translation) from the tropical hypersurface $\mathcal{T}(X)$. Vertices of $Q$ correspond bijectively to connected components of $N_{\mathbb{Q}} \backslash \mathcal{T}(X)$. Let $u \in N_{\mathbb{Q}}$ be a general vector in such a component. We compute the corresponding vertex, which is face $_{u}(Q)$, using the 
following formula from $\left[6\right.$, Theorem 2.2]. Consider the halflines $L_{i}=u+\mathbb{Q} \geq 0 e_{i}$ where $\left\{e_{1}, \ldots, e_{n}\right\}$ is some fixed basis of $N$. Each halfline $L_{i}$ intersects $\mathcal{T}(X)$ in finitely many points $v$, and with each of these we associate the nonnegative integer

$$
\left[N: \mathbb{Z}_{i}+\left(\mathbb{L}_{w} \cap N\right)\right] \cdot m_{v} .
$$

The $i$-th coordinate of the vertex is the sum of these integers, for all $v \in L_{i} \cap \mathcal{T}(X)$.

\section{Complete Intersections and Mixed Volumes}

In this section we study the case when $X$ is a generic complete intersection in a torus $\mathbb{T}=\mathbb{T}^{n}$. Our tropical approach recovers and refines the elimination theory based on Newton polytopes which was developed by Khovanskii and Esterov [13].

Notation 4.1. Let $P_{1}, \ldots, P_{c}$ be lattice polytopes in $M_{\mathbb{Q}}$. Let $P:=P_{1}+\cdots+P_{c}$ be their Minkowski sum and write $P_{I}:=\sum_{i \in I} P_{i}$ for any $I \subseteq\{1,2, \ldots, c\}$. We shall always assume that $\operatorname{dim} P=n$. The normal fan $\mathcal{N}$ of $P$ is a complete fan in $N_{\mathbb{Q}}$.

For each $i=1, \ldots, c$, let $f_{i}$ be a Laurent polynomial in $k[\mathbb{T}]$ whose Newton polytope equals $P_{i}$ and whose coefficients are generic subject to this property. We are interested in the generic complete intersection $X=\left\{f_{1}=\cdots=f_{c}=0\right\}$ in $\mathbb{T}$.

Remark 4.2. The variety $X$ need not be irreducible because the $P_{i}$ can be onedimensional even if $n \geq 2$. This poses some notational difficulties since irreducibility of $X$ was assumed in the previous two sections. However, as we shall see, all irreducible (= connected) components of $X$ have exactly the same tropicalization and multiplicities. Thus by $\mathcal{T}(X)$ we will mean the tropicalization of any irreducible component, with multiplicities multiplied by the number of components. This is consistent with the interpretation of the tropical variety as a cohomology class.

As before in Example 3.16, for any $w \in N_{\mathbb{Q}}=\operatorname{Hom}\left(M_{\mathbb{Q}}, \mathbb{Q}\right)$ and any polytope $Q$, we write face $_{w}(Q)$ for the face of $Q$ at which $w$ attains its minimum. Note that

$$
\text { face }_{w}\left(P_{I}\right)=\sum_{i \in I} \operatorname{face}_{w}\left(P_{i}\right) .
$$

Theorem 4.3 ([20, Prop. 2.4]). The tropical variety $\mathcal{T}(X)$ is supported on a subfan of the normal fan $\mathcal{N}$ of the Minkowski sum $P=P_{1}+\cdots+P_{c}$. A point $w \in N_{\mathbb{Q}}$ lies in $\mathcal{T}(X)$ if and only if the polytope (4.13) has dimension $\geq|I|$ for any $I \subseteq\{1,2, \ldots, c\}$.

For $w=0$ we obtain the following criterion for when the variety $X$ is non-empty:

Corollary 4.4. $X$ is not empty if and only if $\operatorname{dim} P_{I} \geq|I|$ for every $I \subseteq\{1,2, \ldots, c\}$.

In [20] we proved Theorem 4.3 using combinatorial convexity. Here we present an alternative derivation using following general lemma, which is a mixed version of the Kodaira-Fujita Lemma [14, VI.2.16] that characterizes big and nef line bundles.

Lemma 4.5. Let $L_{1}, L_{2}, \ldots, L_{c}$ be globally generated line bundles on a c-dimensional integral scheme $Z$. For $K \subseteq\{1, \ldots, c\}$ let $Z_{K}$ be the image of $Z$ under the map given by $\otimes_{i \in K} L_{i}$. Then $L_{1} \cdot L_{2} \cdot \ldots \cdot L_{c} \geq 0$ and this number is positive if and only if

$$
\operatorname{dim} Z_{K} \geq|K| \text { for any } K \subseteq\{1, \ldots, c\} .
$$


Proof. By the Projection Formula [14, VI.2.11], we can replace $Z$ with its resolution of singularities. So, we may assume that $Z$ is smooth. By Bertini's Theorem, we can choose smooth divisors $E_{i} \in\left|L_{i}\right|$ that intersect transversally. The number of intersection points equals our intersection number $L_{1} \cdot \ldots L_{c}$. In particular, the number is nonnegative and it is positive if and only if $E_{1} \cap \cdots \cap E_{c} \neq \emptyset$. The condition (4.14) is necessary because $\operatorname{dim} Z_{K}<|K|$ implies $\cap_{i \in K} E_{i}=\emptyset$ for general divisors $E_{i} \in\left|\mathcal{L}_{i}\right|$. Now assume (4.14) is satisfied. We use induction on $c$. The case $c=1$ is obvious. Assume that $c>1$. Let $x \in Z$ be a general point. By Bertini's Theorem, we can assume that $x$ lies in a smooth divisor from $\left|\mathcal{L}_{c}\right|$. Let $Z^{\prime}$ be the connected component of $Z$ that contains $x$. It suffices to check the condition (4.14) for $Z^{\prime}$ and all subsets $K \subseteq\{1, \ldots, c-1\}$. Arguing by contradiction, suppose that $\operatorname{dim} Z_{K}^{\prime}<|K|$ for some $K$. Let $F$ be the irreducible component of the fiber of $Z \rightarrow Z_{K}$ that passes through $x$. Then $\operatorname{dim}\left(F \cap Z^{\prime}\right) \geq c-|K|$. By our assumptions (and genericity of $x$ ), it follows that $\operatorname{dim} F=c-|K|$ and $F \subset Z^{\prime}$. As this holds for any component $Z^{\prime}$, the map $Z \rightarrow Z_{\{c\}}$ given by $\mathcal{L}_{c}$ contracts $F$ to a point. Therefore, $F$ is contained in a fiber of the map $Z \rightarrow Z_{K} \times Z_{\{c\}}$. Since the image of $\left|\mathcal{L}_{K}\right| \otimes\left|\mathcal{L}_{\{c\}}\right|$ in $\left|\mathcal{L}_{K \cup\{c\}}\right|$ is a base-point-free linear subsystem, $F$ is contained in a fiber of the map $Z \rightarrow Z_{K \cup\{c\}}$. This contradicts our assumption that $\operatorname{dim} Z_{K \cup\{c\}} \geq|K|+1$.

Proof of Theorem 4.3. Let $A_{i}$ be the set of monomials in $f_{i}$, each regarded as a lattice point in $P_{i}$, and consider the morphism $\mathbb{T} \rightarrow \mathbb{P}^{\left|A_{i}\right|-1}$ defined by these monomials. Let $\tilde{\mathcal{N}}$ be a strictly simplicial refinement of $\mathcal{N}$. There is a sequence of morphisms

$$
\mathbb{P}(\tilde{\mathcal{N}}) \rightarrow \mathbb{P}(\mathcal{N}) \rightarrow \mathbb{P}^{\left|P_{i}\right|-1}-\rightarrow \mathbb{P}^{\left|A_{i}\right|-1},
$$

where $\mathbb{P}^{\left|P_{i}\right|-1} \rightarrow \mathbb{P}^{\left|A_{i}\right|-1}$ is a linear projection. We have similar toric morphisms

$$
\mathbb{P}(\tilde{\mathcal{N}}) \rightarrow \mathbb{P}(\mathcal{N}) \rightarrow \mathbb{P}^{\left|P_{K}\right|-1} \quad \text { for any } K \subseteq\{1, \ldots, c\} .
$$

The toric variety $\mathbb{P}(\tilde{\mathcal{N}})$ is smooth and its toric boundary $\bigcup_{i=1}^{r} D_{i}$ has simple normal crossings. Consider the hypersurface $E_{i}:=\left\{f_{i}=0\right\} \subset \mathbb{T}$ and its closure $\bar{E}_{i}$ in $\mathbb{P}(\tilde{\mathcal{N}})$. Let $\left\{\bar{E}_{i}^{\alpha}\right\}_{\alpha \in J_{i}}$ be the irreducible components of $\bar{E}_{i}$. Then $\bar{E}_{i}$ is a pullback of a general hyperplane divisor in $\mathbb{P}^{\left|A_{i}\right|-1}$ for the morphism (4.15). It follows from Bertini's Theorem that each $\bar{E}_{i}$ is smooth, $E_{i}^{\alpha} \cap E_{i}^{\beta}=\emptyset$ for $\alpha \neq \beta$, and the union of all divisors (both of toric type $D_{i}$ and of type $\bar{E}_{i}^{\alpha}$ ) has simple normal crossings.

Recall that our very affine variety $X$ is the intersection $\bigcap_{i=1}^{c} E_{i}$ in $\mathbb{T}$. It follows that its closure $X(\tilde{\mathcal{N}})$ in $\mathbb{P}(\mathcal{N})$ is smooth, of codimension $c$, and intersects toric boundary divisors $D_{1}, \ldots, D_{r}$ transversally. It follows that the multiplication map

$$
\mathbb{T} \times X(\tilde{\mathcal{N}}) \rightarrow \mathbb{P}(\tilde{\mathcal{N}})
$$

is smooth, and in particular flat. By Theorem 2.12, the tropicalization of any irreducible component $X^{\prime}$ of $X$ is equal to the union of cones of $\tilde{\mathcal{N}}$ that correspond to $c$-dimensional toric strata $Z$ which intersect the closure $X^{\prime}(\tilde{\mathcal{N}})$ of $X^{\prime}$ in $\mathbb{P}(\tilde{\mathcal{N}})$.

Let $Z$ be such a toric stratum and $w$ any vector in the relative interior of the corresponding cone in $\tilde{\mathcal{N}}$. Lemma 4.5 states that $X^{\prime}(\tilde{\mathcal{N}})$ intersects the toric stratum $Z$ if and only if the image of $Z$ under the morphism given by the sum of divisors $\sum_{i \in K} \bar{E}_{i}$ has dimension at least $|K|$ for all $K \subseteq\{1, \ldots, c\}$. This image is the toric 
stratum in the toric variety of $P_{K}$ corresponding to face ${ }_{w}\left(P_{K}\right)$. We conclude that $w$ lies in $\mathcal{T}\left(X^{\prime}\right)=\mathcal{T}(X)$ if and only if $\operatorname{dim}\left(\right.$ face $\left._{w}\left(P_{K}\right)\right)$ is at least $|K|$, for all $K$.

We now describe the multiplicities on the tropical complete intersection $\mathcal{T}(X)$ specified by $P_{1}, \ldots, P_{c}$. The following theorem is the main result in this section.

Theorem 4.6. The multiplicity of $\mathcal{T}(X)$ at a regular point $w$ is the mixed volume

$$
m_{w}=\operatorname{MV}\left(\text { face }_{w}\left(P_{1}\right), \text { face }_{w}\left(P_{2}\right), \ldots, \text { face }_{w}\left(P_{c}\right)\right) .
$$

Here the volume is normalized with respect to the sublattice of $M$ orthogonal to $\mathbb{L}_{w}$.

Proof. We fix a tropical fan on $\mathcal{T}(X)$ which refines the restriction of the normal fan $\mathcal{N}$ of $P_{1}+\cdots+P_{c}$. Since both sides of the equation (4.17) are locally constant on the regular points $w$ of $\mathcal{T}(X)$, it suffices to prove (4.17) only for points $w \in \mathcal{T}(X)$ that lie in the relative interior of a maximal cone $\Gamma$ of the chosen tropical fan structure.

Let $\mathbb{T}_{\Gamma}$ be the $c$-dimensional torus corresponding to $M_{\Gamma}$. The polytope face ${ }_{w}\left(P_{i}\right)$ is the Newton polytope of the initial form $\operatorname{in}_{w}\left(f_{i}\right)$. Consider the subscheme

$$
X_{w}:=\left\{\operatorname{in}_{w}\left(f_{1}\right)=\cdots=\operatorname{in}_{w}\left(f_{c}\right)=0\right\} \subset \mathbb{T} .
$$

The equations $\operatorname{in}_{w}\left(f_{i}\right)$ are equivariant under the action of $\operatorname{Ker}\left[\mathbb{T} \rightarrow \mathbb{T}_{\Gamma}\right]$ and therefore we can regard (4.18) as a system of $c$ equations on $\mathbb{T}_{\Gamma}$ defining a zero-dimensional subscheme $\tilde{X}_{w}$ of $\mathbb{T}_{\Gamma}$. By Bernstein's Theorem [1], the length of the generic complete intersection $\tilde{X}_{w}$ equals the mixed volume on the right hand side of (4.17).

We claim that $\tilde{X}_{w}$ coincides with the scheme-theoretic intersection of $X(\mathcal{N})$ with the toric stratum $Z$ of $\mathbb{P}(\mathcal{N})$ that corresponds to $\Gamma$. Indeed, since $X(\mathcal{N})$ does not intersect strata of $\mathbb{P}(\mathcal{N})$ of codimension $c+1$, the intersection $X(\mathcal{N}) \cap Z$ lies in the open orbit in $Z$. That open orbit is isomorphic to $\mathbb{T}_{\Gamma}$ and the defining equations of $X(\mathcal{N}) \cap Z$ are precisely the $w$-initial forms of the $f_{i}$. Definition 3.1 now implies

$$
m_{w}=\operatorname{length}(X(\mathcal{N}) \cap Z)=\operatorname{length}\left(\tilde{X}_{w}\right) .
$$

With this, the conclusion of the previous paragraph now completes the proof.

Remark 4.7. The multiplicity formula (4.17) makes sense for all $w \in N_{\mathbb{Q}}$ since the right hand side is zero unless the condition of Theorem 4.3 holds. Thus, $\mathcal{T}(X)$ is characterized as the set of all $w$ for which the mixed volume in (4.17) is nonzero.

We now apply our elimination theory of Section 3 to the generic complete intersection $X$. The goal is to compute the image of $X$ under the morphism $\alpha: \mathbb{T}^{n} \rightarrow \mathbb{T}^{d}$ given by a linear map $\mathbf{A}: \mathbb{Z}^{n} \rightarrow \mathbb{Z}^{d}$. We assume that the induced map $f: X \rightarrow Y$ is generically finite of degree $\delta$. This assumption implies in particular that $d \geq n-c$. The tropical variety of $Y=\alpha(X)$ in $\mathbb{Q}^{d}$ is characterized by the following formula.

Corollary 4.8. The tropicalization of the image $Y=\alpha(X)$ is the image of $\mathcal{T}(X)$ under the linear map $\mathbf{A}: \mathbb{Q}^{n} \rightarrow \mathbb{Q}^{d}$. Its multiplicity function is given by the formula

(4.19) $m_{v}=\frac{1}{\delta} \sum_{w \in \mathbf{A}^{-1}(v)} \operatorname{MV}\left(\operatorname{face}_{w}\left(P_{1}\right), \ldots\right.$, face $\left._{w}\left(P_{c}\right)\right) \cdot \operatorname{index}\left(\mathbb{L}_{v} \cap \mathbb{Z}^{d}: \mathbf{A}\left(\mathbb{L}_{w} \cap \mathbb{Z}^{n}\right)\right)$,

for all points $v \in \mathcal{T}(Y)^{0}$ such that $\mathbf{A}^{-1}(v) \cap \mathcal{T}(X)$ is finite and contained in $\mathcal{T}(X)^{0}$. 
Proof. This follows by combining Theorems 1.1 and 4.6.

These results will be of particular interest for applications when $d=n-c+1$. In this case $Y=\alpha(X)$ is a hypersurface in the torus $\mathbb{T}^{d}$, and one wishes to compute the irreducible Laurent polynomial $g \in k\left[\mathbb{T}^{d}\right]$ which vanishes on $Y$. Using Corollary 4.8 we obtain the tropical hypersurface $\mathcal{T}(Y)$ in $\mathbb{Q}^{d}$ together with its multiplicities. By applying the technique described in Example 3.17, we can construct the Newton polytope $Q$ of the unknown Laurent polynomial $g$. Knowledge of its Newton polytope $Q$ greatly facilitates the computation of $g$ from the given equations of $X$. See Example 1.3 for an illustration of how this works when $n=3, c=2$ and $d=2$.

A construction of the Newton polytope $Q$ from the given Newton polytopes $P_{1}, \ldots, P_{c}$ and the linear map $\mathbf{A}$ was recently given by Khovanskii and Esterov [13]. Their construction is based on what they call mixed fiber bodies. These turn out to be equivalent to the mixed fiber polytopes introduced earlier by McMullen [15]. This representation constitutes a geometric refinement of Corollary 4.8, and it leads to a simplified derivation (in Theorem 4.9 below) of the main results in [13].

Let $\pi$ be the canonical map from the lattice $\mathbb{Z}^{n}$ onto $\operatorname{coker}\left(\mathbf{A}^{T}\right)=\mathbb{Z}^{n} / \operatorname{ker}(\mathbf{A})^{\perp}$. We use the same letter $\pi$ also for the induced map of vector spaces $\mathbb{Q}^{n} \rightarrow \operatorname{coker}\left(\mathbf{A}^{T}\right)_{\mathbb{Q}}$. The kernel of $\pi$ is the vector space dual to $\operatorname{ker}(\mathbf{A})$, and we identify it with $\mathbb{Q}^{d}$ above.

Consider the Minkowski sum $P_{\lambda}=\lambda_{1} P_{1}+\cdots+\lambda_{c} P_{c}$ where $\lambda=\left(\lambda_{1}, \ldots, \lambda_{c}\right)$ is a vector of positive unknowns. The map $\pi$ projects $P_{\lambda}$ onto the $(n-d)$-dimensional polytope $\pi\left(P_{\lambda}\right)$. Almost all fibers of this map are polytopes of dimension $d$, and, by integrating them in the Minkowski sense of [3], we obtain a $d$-dimensional fiber polytope $\Sigma_{\pi}\left(P_{\lambda}\right) \subset \operatorname{ker}(\pi)=\mathbb{Q}^{d}$. In fact, $\Sigma_{\pi}\left(P_{\lambda}\right)$ is a homogeneous polynomial of degree $n-d+1$ whose coefficients are certain lattice polytopes $Q_{i_{1} i_{2} \cdots i_{c}}$ in $\mathbb{Q}^{d}$ :

$$
\Sigma_{\pi}\left(P_{\lambda}\right)=\Sigma_{\pi}\left(\lambda_{1} P_{1}+\cdots+\lambda_{c} P_{c}\right)=\sum_{i_{1}+\cdots+i_{c}=n-d+1} \lambda_{1}^{i_{1}} \lambda_{2}^{i_{2}} \cdots \lambda_{c}^{i_{c}} \cdot Q_{i_{1} i_{2} \cdots i_{c}} .
$$

This decomposition is due to McMullen [15], and it was rediscovered in [13, §3]. Now, if $n-d+1=c$ then the formula (4.20) defines the mixed fiber polytope

$$
\Sigma_{\pi}\left(P_{1}, P_{2}, \ldots, P_{c}\right):=Q_{11 \cdots 1} .
$$

It is this polytope which is computed by our Corollary 4.8 in the hypersurface case:

Theorem 4.9 (Khovanskii and Esterov [13]). If $c=n-d+1$ then the Newton polytope of the hypersurface $Y=\alpha(X) \subset \mathbb{T}^{d}$ equals the mixed fiber polytope (4.21).

Proof. We consider the Minkowski weight [9] of codimension $c$ defined by the $n$ dimensional polytope $\lambda_{1} P_{1}+\cdots+\lambda_{c} P_{c}$. As a tropical variety, this is the union of the cones of codimension $\geq c$ in the normal fan of this polytope, with weights

$$
w \mapsto \operatorname{Vol}\left(\operatorname{face}_{w}\left(P_{1}\right)+\cdots+\text { face }_{w}\left(P_{c}\right)\right) .
$$

Here Vol is the normalized $c$-dimensional volume on $\mathbb{L}_{w}^{\perp}$. The image in $\operatorname{ker}(\pi)^{*}=\mathbb{Q}^{d}$ of this $c$-dimensional fan under the linear map $\mathbf{A}$ is the union of the codimension one cones in the normal fan of the fiber polytope $\Sigma_{\pi}\left(\lambda_{1} P_{1}+\cdots+\lambda_{c} P_{c}\right)$. It supports the Minkowski weight of codimension 1 of $\Sigma_{\pi}\left(\lambda_{1} P_{1}+\cdots+\lambda_{c} P_{c}\right)$, which is given by

$$
v \mapsto \sum_{w \in \mathbf{A}^{-1}(v)} \operatorname{Vol}\left(\lambda_{1} \operatorname{face}_{w}\left(P_{1}\right)+\cdots+\lambda_{c} \text { face }_{w}\left(P_{c}\right)\right) \cdot \operatorname{index}\left(\mathbb{L}_{v} \cap \mathbb{Z}^{d}: \mathbf{A}\left(\mathbb{L}_{w} \cap \mathbb{Z}^{n}\right)\right) .
$$


This expression is a homogeneous polynomial of degree $c$ in the unknowns $\lambda_{1}, \ldots, \lambda_{c}$. The coefficient of the squarefree monomial $\lambda_{1} \lambda_{2} \cdots \lambda_{c}$ in this polynomial equals the right hand side of (4.19), up to the global constant $\delta$. This proves Theorem 3.2 in [13], which states that the mixed fiber body actually exists as a polytope, and we conclude that $(1 / \delta) \cdot \Sigma_{\pi}\left(P_{1}, P_{2}, \ldots, P_{c}\right)$ equals the Newton polytope of the hypersurface $Y$.

Example 4.10. In Example 1.3 we are given two tetrahedra in three-space, namely $P_{1}=\operatorname{conv}\left\{0,3 e_{1}, 3 e_{2}, 3 e_{3}\right\}$ and $P_{2}=\operatorname{conv}\left\{0,-2 e_{1},-2 e_{2},-2 e_{3}\right\}$. Their Minkowski sum $P_{1}+P_{2}$ is a 3 -polytope with 12 vertices, 24 edges and 14 facets. The map $\pi: \mathbb{Q}^{3} \rightarrow \mathbb{Q}^{1}$ is given in coordinates by $(x, y, z) \mapsto x-2 y+z$. The fiber polytope

$$
\Sigma_{\pi}\left(\lambda_{1} P_{1}+\lambda_{2} P_{2}\right)=\lambda_{1}^{2} \cdot Q_{20}+\lambda_{1} \lambda_{2} \cdot Q_{11}+\lambda_{2}^{2} \cdot Q_{02}
$$

is a polygon with ten vertices. Its summands $Q_{20}$ and $Q_{02}$ are quadrangles, while the mixed fiber polytope $Q_{11}=\Sigma_{\pi}\left(P_{1}, P_{2}\right)$ is a hexagon, affinely isomorphic to (1.5).

Example 4.11. The Newton polytopes of resultants in [19] are mixed fiber polytopes. Here $f_{1}, \ldots, f_{c}$ are Laurent polynomials in $c-1$ variables whose coefficients are distinct unknowns. See also $[6, \S 6]$ for the case of fewer than $c-1$ variables.

We close this section with the remark that (ordinary) fiber polytopes are special instances of mixed fiber polytopes. Suppose that $P_{1}=P_{2}=\cdots=P_{c}$ are all equal to the same fixed polytope $P$ in $\mathbb{Q}^{n}$. Then the polytope $\Sigma_{\pi}\left(P_{\lambda}\right)$ in (4.20) equals

$$
\Sigma\left(\lambda_{1} P_{1}+\cdots+\lambda_{c} P_{c}\right)=\left(\lambda_{1}+\cdots+\lambda_{c}\right)^{c} \cdot \Sigma_{\pi}(P) \subset \mathbb{Q}^{d} .
$$

Hence the mixed fiber polytope $\Sigma_{\pi}(P, \cdots, P)$ is the fiber polytope $\Sigma_{\pi}(P)$ times $1 / c !$.

We conclude that the study of fiber polytopes, which is an active area in geometric combinatorics, can be regarded as a very special case of tropical elimination theory.

Corollary 4.12. If $X$ is a generic complete intersection in $\mathbb{T}^{n}$ defined by $n-d+1$ Laurent polynomials that share the same full-dimensional Newton polytope $P$, then the Newton polytope of the hypersurface $Y=\alpha(X) \subset \mathbb{T}^{d}$ is the fiber polytope $\Sigma_{\pi}(P)$.

\section{Tropical Implicitization}

We now turn our attention to the problem of implicitization [20]. Let $f_{1}, \ldots, f_{s}$ be Laurent polynomials in unknowns $t_{1}, \ldots, t_{r}$ and consider the rational map

$$
f=\left(f_{1}, \ldots, f_{s}\right): \mathbb{T}^{r} \rightarrow \mathbb{T}^{s}
$$

defined by these Laurent polynomials. Let $Y$ be the closure of the image of $f$ in $\mathbb{T}^{s}$. For simplicity, we assume from the beginning that the fiber of $f$ over a generic point of $Y$ is finite, and consists of $\delta$ points. Computing $Y$ is generally a hard problem in computer algebra. Our goal is to compute instead the tropical variety $\mathcal{T}(Y)$, with the aim of recovering (information about) the variety $Y$ from its tropicalization $\mathcal{T}(Y)$.

Let $P_{1}, \ldots, P_{s} \subset \mathbb{Q}^{r}$ be the Newton polytopes of $f_{1}, \ldots, f_{s}$. We first consider the generic case, when $f_{i}$ has generic coefficients relative to its Newton polytope $P_{i}$, and later we address the case when the $f_{i}$ have special coefficients. A rule for computing $\mathcal{T}(Y)$ from $P_{1}, \ldots, P_{s}$ was stated (with proof) in [20, Theorem 2.1], and a formula 
for the multiplicities on $\mathcal{T}(Y)$ was stated (without proof) in [20, Theorem 4.1]. In Theorem 5.1 below we give a complete derivation for both of these results. Let

$$
\Psi=\left(\Psi_{1}, \ldots, \Psi_{s}\right): \mathbb{Q}^{r} \rightarrow \mathbb{Q}^{s}
$$

be the tropicalization of the map $f$. Its $i$ th coordinate $\Psi_{i}(w)=\min \left\{w \cdot v: v \in P_{i}\right\}$ is the support function of $P_{i}$. The image of $\Psi$ is contained in $\mathcal{T}(Y)$ but this containment is usually strict. Our construction will explain the set difference $\mathcal{T}(Y) \backslash \operatorname{image}(\Psi)$.

Let $C$ be any cone in the normal fan of the Minkowski sum $P=P_{1}+\cdots+P_{s}$ and $J$ any subset of $\{1, \ldots, s\}$. Consider the sublattice of $\mathbb{Z}^{s}$ spanned by $\Psi\left(C \cap \mathbb{Z}^{r}\right)+\mathbb{Z}^{J}$. If the rank of this sublattice is $r$ then index $(C, J)$ denotes its index in the maximal rank $r$ sublattice of $\mathbb{Z}^{s}$ that contains it. Otherwise we set $\operatorname{index}(C, J)=0$. Write face $_{C}\left(P_{j}\right)$ for the face of $P_{j}$ at which the linear forms in the relative interior of $C$ are minimized. The $|J|$-dimensional normalized mixed volume

$$
\operatorname{MV}\left(\operatorname{face}_{C}\left(P_{j}\right): j \in J\right)
$$

is positive if and only if $\operatorname{dim}\left(\sum_{i \in K}\right.$ face $\left._{C}\left(P_{i}\right)\right) \geq|K|$ for all subsets $K \subseteq J$.

Theorem 5.1. The tropical variety $\mathcal{T}(Y)$ is the union of the cones $\Psi(C)+\mathbb{R}_{\geq 0}^{J}$ over all pairs $(C, J)$ such that (5.22) is positive. The multiplicity $m_{w}$ at any regular point $w$ of $\mathcal{T}(Y)$ is the sum of the scaled mixed volumes

$$
\frac{1}{\delta} \cdot \operatorname{index}(C, J) \cdot \operatorname{MV}\left(\operatorname{face}_{C}\left(P_{j}\right): j \in J\right)
$$

where $(C, J)$ runs over all pairs such that $\Psi(C)+\mathbb{R}_{\geq 0}^{J}$ contains $w$.

Proof. The graph of the map $f$ is a generic complete intersection of codimension $s$ in $\mathbb{T}^{r+s}$. Namely, writing $\left(t_{1}, \ldots, t_{r}, y_{1}, \ldots, y_{s}\right)$ for the coordinates on $\mathbb{T}^{r+s}$, by the graph of $f$ we mean the subvariety $X$ of $\mathbb{T}^{r+s}$ which is defined by the equations

$$
f_{1}(t)-y_{1}=f_{2}(t)-y_{2}=\cdots=f_{s}(t)-y_{s}=0 .
$$

These are $s$ generic Laurent polynomials in $r+s$ unknowns. Their Newton polytopes in $\mathbb{Q}^{r} \oplus \mathbb{Q}^{s}$ have the form $P_{i}^{\prime}:=\operatorname{conv}\left(P_{i} \cup\left\{e_{i}\right\}\right)$ for $i=1,2, \ldots, s$, where $e_{1}, \ldots, e_{s}$ are the standard basis vectors of $\mathbb{Q}^{s}$. We shall apply the results of Section 4 (with $d=s$ and $n=s+t$ ) to the polytopes $P_{1}^{\prime}, \ldots, P_{s}^{\prime}$ and to the linear map A which takes vectors $(u, v)$ in $\mathbb{Q}^{r} \oplus \mathbb{Q}^{s}$ to their second component $v \in \mathbb{Q}^{s}$. The corresponding homomorphism of tori is $\alpha: \mathbb{T}^{r+s} \rightarrow \mathbb{T}^{s},(t, y) \mapsto y$ and we have $Y=\alpha(X)$.

The tropical variety $\mathcal{T}(Y)$ equals the set of all vectors $v \in \mathbb{Q}^{s}$ such that

$$
\operatorname{MV}\left(\operatorname{face}_{(u, v)}\left(P_{1}^{\prime}\right), \ldots, \text { face }_{(u, v)}\left(P_{s}^{\prime}\right)\right)
$$

is positive for some $u \in \mathbb{Q}^{r}$. Suppose this is the case where $(u, v)$ is a regular point of $\mathcal{T}(X)$. Let $C$ be the cone in the normal fan of $P$ which contains $u$ in its relative interior. The vector $v-\Psi(u)$ is non-negative, for if its $i$-th coordinate were negative then face $_{(u, v)}\left(P_{i}^{\prime}\right)$ would be a point $\left\{e_{i}\right\}$. Let $J$ be the set of all indices $j$ with $v_{j}>\Psi_{j}(u)$. For $j \in J$ we have $\operatorname{face}_{(u, v)}\left(P_{j}^{\prime}\right)=$ face $_{u}\left(P_{j}\right)=$ face $_{C}\left(P_{j}\right)$. For $i \in$ $\{1, \ldots, s\} \backslash J$, face $_{v}\left(P_{i}\right)$ must be a single point $p_{i}$ in $\mathbb{Q}^{r}$, since $(u, v)$ is regular on $\mathcal{T}(X)$, and hence face $_{(u, v)}\left(P_{i}^{\prime}\right)$ is the primitive line segment with vertices $p_{i}$ and 
$e_{i}$. Since the lattice spanned by these segments is a direct summand of $\mathbb{Z}^{r+s}$, the normalized mixed volume in (5.24) remains unchanged if we extract these segments:

$$
\operatorname{MV}\left(\operatorname{face}_{(u, v)}\left(P_{1}^{\prime}\right), \ldots, \operatorname{face}_{(u, v)}\left(P_{s}^{\prime}\right)\right)=\operatorname{MV}\left(\operatorname{face}_{C}\left(P_{j}\right): j \in J\right) .
$$

The linear space $\mathbb{L}_{(u, v)}$ for the neighborhood of $(u, v)$ on $\mathcal{T}(X)^{0}$ is spanned by the vectors $\left(u^{\prime}, v^{\prime}\right)$ where $u^{\prime}$ runs over $C$ and $v^{\prime}$ satisfies $v_{i}^{\prime}=\Psi\left(u^{\prime}\right)_{i}$ for $i \in\{1, \ldots, s\} \backslash J$. This implies that $\mathbf{A}\left(\mathbb{L}_{(u, v)} \cap \mathbb{Z}^{r+s}\right)$ equals the lattice generated by $\Psi\left(C \cap \mathbb{Z}^{r}\right)+\mathbb{Z}^{J}$. The maximal rank $r$ sublattice of $\mathbb{Z}^{s}$ that contains this lattice is $\mathbb{L}_{v} \cap \mathbb{Z}^{s}$. We conclude

$$
\left[\mathbb{L}_{v} \cap \mathbb{Z}^{s}: \mathbf{A}\left(\mathbb{L}_{(u, v)} \cap \mathbb{Z}^{r+s}\right)\right]=\operatorname{index}(C, J) .
$$

Theorem 5.1 now follows by combining (5.25) and (5.26) with Corollary 4.8.

Example 5.2. Consider the matrix $\left(m_{i j}\right)=\left[\begin{array}{cccccc}1 & 0 & 0 & 0 & 1 & 1 \\ 0 & 1 & 0 & 1 & 0 & -1 \\ 0 & 0 & 1 & -1 & -1 & 0\end{array}\right]$ and let $f: \mathbb{T}^{3} \rightarrow \mathbb{T}^{6}$ be a rational map defined by six Laurent polynomials, with generic coefficients, with Newton polytopes given by the six parallelopipeds

$$
P_{i}=\left[m_{1 i}, 2\right] \times\left[m_{2 i}, 2\right] \times\left[m_{3 i}, 2\right] .
$$

Let $Y=\overline{f\left(\mathbb{T}^{3}\right)}$. A simple calculation using Theorem 5.1 (that can be performed using the software $\operatorname{Tr} \operatorname{Im}[11]$ ) shows that $\mathcal{T}(Y)$ contains the cone $C$ which is spanned by $e_{1}, e_{2}$, and $e_{3}$, and the only other cone intersecting its interior is the cone $D$ spanned by rows of the matrix $\left(m_{i j}\right)$. Moreover, $C \cap D$ is the ray spanned by $e_{1}+e_{2}+e_{3}$. It follows that the set of regular points of $\mathcal{T}(Y)$ is not convex. In particular, there does not exist a coarsest fan supported on $\mathcal{T}(Y)$.

We now discuss the implicitization problem for maps $f$ which are not necessarily generic. The $s$ hypersurfaces $E_{i}:=\left\{f_{i}=0\right\} \subset \mathbb{T}^{r}$ are assumed to be reduced, irreducible, and different. We focus on computing $\mathcal{T}(Y)$ as a set, leaving the question of multiplicities for future work. Let $X:=\mathbb{T}^{r} \backslash \cup_{i=1}^{s} E_{i}$, so that $f$ induces a morphism $X \rightarrow Y \subset \mathbb{T}^{s}$. We can thus apply Theorem 2.9 to compute $\mathcal{T}(Y)$. We introduce the following notation and terminology. For any compactification $X \subset \bar{X}$ and any irreducible boundary divisor $D$, let $[D]$ be the vector $\left[\operatorname{val}_{D}\left(f_{1}\right), \ldots, \operatorname{val}_{D}\left(f_{s}\right)\right] \in \mathbb{Q}^{s}$. By Theorem 2.4, $[D] \in \mathcal{T}(Y)$ for any $D$. We define the simplicial complex $\Delta(\bar{X})$ that describes the combinatorics of the boundary of $\bar{X}$ as in Theorem 2.6. As in Remark 2.7, the tropical variety $\mathcal{T}(Y)$ is contained in the union of cones with rays given by $[D]$ and cones described by $\Delta(\bar{X})$. We say that $\bar{X}$ computes $\mathcal{T}(X)$ if $\mathcal{T}(Y)$ is equal to this union of cones. For example, if $\bar{X}$ is smooth with a normally crossing boundary then $\bar{X}$ computes $\mathcal{T}(X)$ by Corollary 2.9. But Proposition 3.9 suggests that it may suffice to assume that $\bar{X}$ has "combinatorial normal crossings", i.e. to assume that $k$ boundary divisors intersect in codimension $k$. Below we describe how this works for surfaces, the case $r=2$. In what follows we assume $r=2$.

We construct a compactification $\bar{X}$ of $X$ in two steps. First we compactify $X$ by a toric surface $\mathbb{P}(\tilde{\mathcal{N}})$, where $\tilde{\mathcal{N}}$ is some strictly simplicial refinement of the normal fan $\mathcal{N}$ of the Minkowski sum of Newton polygons of $f_{1}, \ldots, f_{r}$. By Theorem 2.4, the rays of $\mathcal{T}(Y)$ that we see on this stage are $\left[E_{i}\right]=e_{i}$ for $i=1, \ldots, s$ and $\left[D_{j}\right]=\mathbb{Q}_{\geq 0} \Psi\left(\rho_{j}\right)$, where $\rho_{i} \subset \tilde{\mathcal{N}}$ for $j=1, \ldots, p$ are the rays that correspond to the toric divisors $D_{j}$. 
Consider a morphism $\bar{X} \rightarrow \mathbb{P}(\tilde{\mathcal{N}})$ which is a composition of blow-ups with smooth centers. Let $\tilde{E}_{1}, \ldots, \tilde{E}_{s}, \tilde{D}_{1}, \ldots, \tilde{D}_{p}$ be the proper transforms and $F_{1}, \ldots, F_{q}$ the exceptional divisors. We determine the simplicial complex $\Delta(\bar{X} ; E, D, F)$ as in Theorem 2.6. Let $u_{i j}$ (resp. $v_{i j}$ ) be the coefficient of $F_{i}$ in the pullback of $E_{j}$ (resp. $D_{j}$ ). Note that on $\mathbb{P}(\tilde{\mathcal{N}})$ the divisor of the rational function $f_{i}$ is given by $\left(f_{i}\right)=E_{i}+$ $\sum_{j} \Psi_{i}\left(\rho_{j}\right) D_{j}$. It follows that on $\bar{X}$ we have

$$
\left(f_{i}\right)=\tilde{E}_{i}+\sum_{j} \Psi_{i}\left(\rho_{j}\right) \tilde{D}_{j}+\sum_{k}\left(u_{k i}+\sum_{j} \Psi_{i}\left(\rho_{j}\right) v_{k j}\right) F_{k} .
$$

It follows that the vectors $\left[F_{k}\right]$ in $\mathbb{Q}^{s}$ are given by the formula

$$
\left[F_{k}\right]=\left(u_{k 1}, \ldots, u_{k s}\right)+\Psi\left(v_{k 1} \rho_{1}+\cdots+v_{k p} \rho_{p}\right) .
$$

Proposition 5.3. Let $r=2$ and let $\bar{X} \rightarrow \mathbb{P}\left(\mathcal{N}^{\prime}\right)$ be a morphism, constructed as a composition of blow-ups of points, such that no three divisors from the collection $\tilde{E}$, $\tilde{D}$, and $F$ have a common point. Then $\bar{X}$ computes the tropical surface $\mathcal{T}(Y)$.

Proof. It suffices to observe, using (5.27), that new exceptional divisors added by a resolution of singularities $\bar{X}^{\prime} \rightarrow \bar{X}$ do not change the tropicalization: each time we blow-up a point $p$ that belongs to a unique boundary divisor $\Delta$, we obviously have $\left[F_{p}\right]=[\Delta]$ and the only new simplex created is a pair $\left\{F_{p}, \Delta\right\}$, which gives rise to the same ray $[\Delta]$ on the tropicalization. Similarly, the blow-up of a point $p$ that belongs to two boundary divisors $\Delta_{1}$ and $\Delta_{2}$ creates a new ray $\left[F_{p}\right]=m_{1}\left[\Delta_{1}\right]+m_{2}\left[\Delta_{2}\right]$, where $m_{i}$ is the multiplicity of $\Delta_{i}$ at $p$, and the tropicalization stays the same.

\section{Acknowledgements}

We are grateful to Igor Dolgachev, Paul Hacking, Sean Keel, David Speyer, and Josephine Yu for useful discussions. Both authors acknowledge partial support by the National Science Foundation (DMS-0456960, DMS-0701191). The second author was also supported by a Sloan Research Fellowship.

\section{References}

[1] D. Bernstein, The number of roots of a system of equations, Functional Analysis and its Applications 9 (1975) 183-185.

[2] R. Bieri and J. R. J. Groves, The geometry of the set of characters induced by valuations, J. Reine Angew. Math. 347 (1984), 168-195

[3] L. Billera and B. Sturmfels, Fiber polytopes, Annals of Math. 135 (1992) 527-549.

[4] T. Bogart, A. Jensen, D. Speyer, B. Sturmfels and R. Thomas, Computing tropical varieties, Journal of Symbolic Computation 42 (2007) 54-73.

[5] N. Bourbaki: Commutative Algebra, Springer, Berlin, 1989.

[6] A. Dickenstein, E.-M. Feichtner and B. Sturmfels, Tropical discriminants, Journal of the American Mathematical Society 20 (2007) 1111-1133.

[7] M. Einsiedler, M. Kapranov, D. Lind, Non-archimedean amoebas and tropical varieties, Journal fur die reine und angewandte Mathematik, 60 (2006) 139-157.

[8] W. Fulton, Intersection theory, Springer Verlag, New York, 1984.

[9] W. Fulton and B. Sturmfels: Intersection theory on toric varieties, Topology 36 (1997) 335-353.

[10] P. Hacking, S. Keel, and J. Tevelev, Stable pair, tropical, and log canonical compact moduli of del Pezzo surfaces, math.AG/0702505. 
[11] B. Sturmfels and J. Yu, Tropical implicitization and mixed fiber polytopes, in M. Stillman, N. Takayama and J. Verschelde (eds.): Software for Algebraic Geometry, I.M.A. Volumes in Mathematics and its Applications, to appear.

[12] E. Katz and S. Payne: Piecewise polynomials, Minkowski weights, and localization on toric varieties, math. AG/0703672.

[13] A. Khovanskii and A. Esterov: Elimination theory and Newton polyhedra, math. AG/0611107.

[14] J. Kollár: Rational Curves on Algebraic Varieties, Springer Verlag, 1996.

[15] P. McMullen, Mixed fibre polytopes, Discrete Comput. Geom. 32 (2004) 521-532.

[16] P. Samuel, À propos du théorème des unités, Bull. Sci. Math. (2) 90, 1966, 89-96.

[17] D. Speyer, Tropical Geometry, Ph.D. Dissertation, UC Berkeley, 2005.

[18] D. Speyer and B. Sturmfels, The tropical Grassmannian, Adv. in Geometry 4 (2004) 389-411.

[19] B. Sturmfels, On the Newton polytope of the resultant, J. Alg Combinatorics 3 (1994) 207-236.

[20] B. Sturmfels, J. Tevelev, and J. Yu, The Newton polytope of the implicit equation, Moscow Mathematical Journal 7 (2007) 327-346.

[21] J. Tevelev, Compactifications of suvbarieties of tori, American Journal of Mathematics 129 (2007) 1087-1104.

Department of Mathematics, University of California, Berkeley, CA 94720, USA

E-mail address: bernd@math.berkeley.edu

Department of Mathematics, University of Massachusetts, Amherst, MA 01003, USA

E-mail address: tevelev@math.umass.edu 